\title{
Study on the Relationship between Sports Skills and Visual Image Operation
}

\author{
Peng Zeng, ${ }^{1}$ Long Liu, ${ }^{2}$ Timon Cheng-Yi Liu, ${ }^{1}$ and Xiang-Bo Yang ${ }^{1,3}$ \\ ${ }^{1}$ College of Physical Education and Sports Science, South China Normal University, University Town, Guangzhou 510006, China \\ ${ }^{2}$ Department of Chemistry and Biology, College of Science, National University of Defense Technology, Changsha 410073, China \\ ${ }^{3}$ MOE Key Laboratory of Laser Life Science and Institute of Laser Life Science, College of Biophotonics, South China Normal University, \\ Guangzhou 510631, China
}

Correspondence should be addressed to Timon Cheng-Yi Liu; liutcy@scnu.edu.cn

Received 27 February 2014; Revised 22 April 2014; Accepted 22 April 2014; Published 6 May 2014

Academic Editor: Quan-Guang Zhang

Copyright (C) 2014 Peng Zeng et al. This is an open access article distributed under the Creative Commons Attribution License, which permits unrestricted use, distribution, and reproduction in any medium, provided the original work is properly cited.

120 college athletes including 30 gymnasts, 30 ball players, 30 athletes in track and field, and 30 swimmers with different levels of sports skills were measured on the operation speed and accuracy of visual images in the present study. The results showed that there was a close relationship between sport skill level and the operation level of visual images. The higher the sport skill level was, the higher the operation level was. The gymnasts were faster in the operation of visual images than the other athletes, but there was no significant difference among athletes of other sports games in visual image operation. The athletes achieved great improvement in their ability of visual image, but there was no significant difference between different genders.

\section{Introduction}

Visual image refers to the general image formed from the visual perception in the brain. There is a correlation between visuospatial working-memory capacity and motor sequence chunk length $[1,2]$. It has been found that the operation of visual image, especially motor image, has been generalized to tasks beyond video-game playing. For example, training in action video games can increase the speed of perceptual processing [3] and cognitive flexibility [4]. On the other hand, some studies have investigated the influence of motor activities, such as baseball playing and tennis playing [5], volleyball playing and rowing [6], archery [7], kung fu [8], and Suryanamaskar [9], on the visual image operation. For example, Kida et al. [5] have studied the visual image operation Go or No go of 82 university students (22 baseball players, 22 tennis players, and 38 nonathletes) and 17 professional baseball players and found that intensive practice improved the reaction time. The present study investigated the reaction time and error of 120 college athletes including 30 gymnasts, 30 ball players, 30 athletes in track and field, and 30 swimmers on the visual image operation.

\section{Methodology}

2.1. Subjects. Altogether 120 college athletes including 30 gymnasts, 30 ball players, 30 athletes in track and field, and 30 swimmers with different sports levels were selected from colleges and universities in Guangdong, China. Three groups with high, medium, and low sports levels were formed with 40 students in each group. And the male and female subjects were balanced (male: female $=1: 1$ ) ranging in age from 18 to 25 years. According to the National Athletes Skills Standard of China, the subjects in the high sports level group reached the first or above sports level, and the subjects in the medium sports level group reached the second sports level. Those in the low sports level group were ordinary college students. In each group, there were 10 gymnasts, 10 ball players, 10 athletes in track and field, and 10 swimmers, with 20 males and 20 females. All the subjects' visual acuity (including the corrected visual acuity) was above 1.0.

2.2. Experimental Material and Measurement. The stimulus variable was two different sets of graphic materials (Figure 1) controlled and displayed on the screen of a microcomputer. 


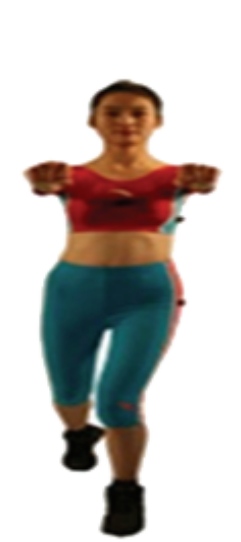

(a)
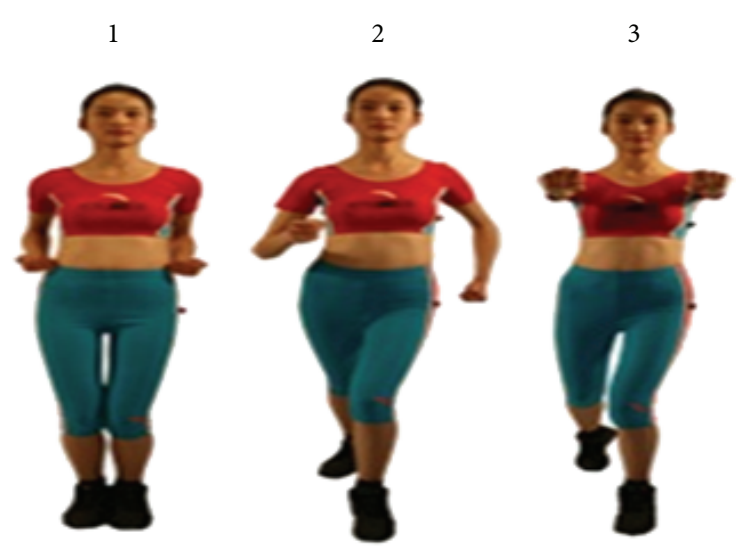

(b)
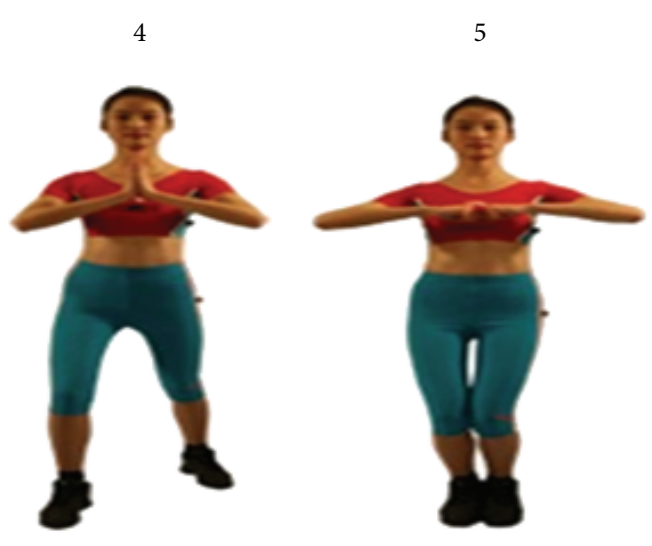

FIGURE 1: One left picture (a) serving as standard stimulus and a set of 5 numbered right pictures (b) serving as selective stimuli.

The response variable consisted of the reaction time and the number of errors made by the subjects in conducting the experimental tasks, which were automatically recorded by the computer.

In the experiment, the subjects visually focused on the screen of the computer with the dominant hand on the keyboard. One picture (Figure 1(a)) serving as standard stimulus was displayed on the computer screen for 2 seconds. After a 5 -second interval, a set of 5 numbered pictures (Figure 1(b)) serving as selective stimuli were displayed. Of the 5 numbered pictures (Figure 1(b)), one was exactly the same as the standard stimulus and the other four were just very similar to the standard stimulus. The subjects were required to make the choice and press the corresponding number on the keyboard as soon as possible. Every subject had 5 trials for practice to be familiar with the operation procedure of the experiment and was then required to take 15 consecutive trials in the test with the reaction time and errors recorded.

All the collected data were processed and analyzed with the help of SPSS 8.0. Data were expressed as mean \pm standard deviation. Independent $t$-tests and one-way ANOVA were carried out, respectively.

\section{Results}

3.1. Results and the Analysis of the Visual Image Operation of Subjects with Different Sports Levels. Table 1 showed the results of the tests, where the operation speed and accuracy of the visual image operation increased with the improvement of the level of motor skills. The higher the level of motor skills was, the shorter the reaction time was and the fewer the errors were, respectively. The reaction time and errors of the high sports level group were shorter and fewer than those of the medium sports level group, and the reaction time and errors of the medium sports level group were shorter and fewer than those of the low sports level group, respectively. One-way ANOVA showed that there was significant difference among the three experimental groups $(F=9.56, P<0.01)$.
TABLE 1: Visual image operation performance of subjects with different sports levels.

\begin{tabular}{lcccc}
\hline \multirow{2}{*}{ Group } & \multicolumn{2}{c}{ Reaction time (s) } & \multicolumn{2}{c}{ Error (times) } \\
& $\mathrm{M}$ & $\mathrm{SD}$ & $\mathrm{M}$ & $\mathrm{SD}$ \\
\hline High sports level & 4.56 & 1.89 & 3.13 & 1.43 \\
Medium sports level & 4.76 & 1.95 & 3.21 & 1.65 \\
Low sports level & 5.23 & 2.13 & 3.87 & 2.01 \\
\hline
\end{tabular}

Notes: $\mathrm{M}$ and SD denoted the mean reaction time and standard deviation, respectively.

TABLE 2: Visual image operation performance of subjects of different gender.

\begin{tabular}{lccccc}
\hline & \multicolumn{2}{c}{ Male } & \multicolumn{2}{c}{ Female } & \multirow{2}{*}{$P$} \\
& M & SD & M & SD & \\
\hline Reaction time (s) & 4.82 & 1.89 & 4.78 & 1.94 & $>0.05$ \\
Error (times) & 3.43 & 1.73 & 3.49 & 1.89 & $>0.05$ \\
\hline
\end{tabular}

Notes: $\mathrm{M}$ and SD denoted the mean reaction time and standard deviation, respectively.

3.2. The Test Results of the Visual Image Operation on Subjects of Different Gender. Table 2 showed that there was no significant difference either on the reaction time or on the errors between the male and female subjects in visual image operation in the present study from independent $t$-tests of the reaction time and error rates.

3.3. The Test Results of the Visual Image Operation on Subjects of Different Sports Games. Independent $t$-tests were carried out with the data in Table 3, showing that gymnasts significantly outperformed the ball players, athletes in track and field, and swimmers on the operation speed. The differences were significant. But the differences among the other three types of athletes on the visual image operation which were indicated through the reaction time and errors were not significant. 
TABLE 3: Visual image operation performance of subjects of different sports games.

\begin{tabular}{lcccccrrr}
\hline & \multicolumn{2}{c}{ Ball players } & \multicolumn{2}{c}{ Gymnasts } & \multicolumn{2}{c}{ Athletes in T and F } & \multicolumn{2}{c}{ Swimmers } \\
& $\mathrm{M}$ & $\mathrm{SD}$ & $\mathrm{M}$ & $\mathrm{SD}$ & $\mathrm{M}$ & $\mathrm{SD}$ & $\mathrm{M}$ & $\mathrm{SD}$ \\
\hline Reaction time (s) & 4.87 & 1.93 & 4.40 & 1.89 & 4.95 & 1.84 & 4.94 \\
Error (times) & 3.33 & 1.56 & 3.21 & 1.45 & 3.35 & 1.38 & 3.01 \\
\hline
\end{tabular}

Notes: $\mathrm{M}, \mathrm{SD}, \mathrm{T}$, and F denoted the mean reaction time, standard deviation, track, and field, respectively.

\section{Discussions}

Kida et al. [5] found that the athletic skill level improved the reaction time of Go or No go visual image operation. The present study further found that the athletic skill level improved the operation level of more complicated visual images. These may play a role in a comprehensive and thorough understanding of the interrelationship between motor learning activity and visual image operation.

4.1. Speed-Specific Homeostasis. In many everyday situations, speed is of the essence. Speed is a function of an organism. There may be a speed-specific homeostasis ( $\mathrm{SeSH}$ ), a negative-feedback response of an organism to maintain the speed-specific internal conditions so that the speed is perfectly performed $[10,11]$. A speed in/far from its SeSH may be called a normal/dysfunction speed. For a normal speed, fast decision means few mistakes. The higher the speed fitness, the faster the decision and the fewer the mistakes. However, for a dysfunctional speed, fast decision means more mistakes. As the physical fitness can be increased by exercise training, the reaction times can be reduced with appropriate training, within one individual, across a range of tasks, and without compromising accuracy. Dye et al. [3] have reviewed evidence that the very act of playing action video games significantly reduces reaction times without sacrificing accuracy. Critically, this increase in speed is observed across various tasks beyond game situations. Video gaming may therefore provide an efficient training regimen to induce a general speeding of perceptual reaction times without decreases in accuracy of performance. Some studies have also found that motor activities such as baseball and tennis playing [5], volleyball playing and rowing [6], kung fu [8], Suryanamaskar [9] and gymnastics, ball playing, games in track and field, and swimming in this paper also significantly reduce reaction times without sacrificing accuracy.

4.2. The Relationship between Motor Skill Level and Visual Image Operation Level. In the present study, our experiment results showed that there were significant differences among the three experimental groups on visual image operation. The high sports level group significantly outperformed the medium sports level group, and the medium sports level group significantly outperformed the low sports level group. This suggested that the visual image of the subjects was closely related to the motor skill level.

Why did the visual image operation level increase with the motor skill level? One reason might be because both of them are the external activities of the same body. The two activities have shared the same internal functions such as the essential heart functions [12]. Bhavanani et al. [9] found that performance of Suryanamaskar produced immediate decrease in both auditory reaction time and visual reaction time, and heart rate increased significantly following Suryanamaskar compared with both self-control and external-control group. Generally, physical fitness can increase action heart rate but decrease rest heart rate [13] and then decrease the reaction time. On the other hand, sport activity is the essential activity of an athlete according to the scale-free network theory [12]. Visual image operation is his/her nonessential activity, but can be learned in an implicit way. Therefore, the longer the sport training is, the higher the athletic skill level is, and then the higher the visual image operation is. However, the implicit learning in sports training has not been widely studied yet.

The deep reason might be the cross-talking between visual nerves and motor nerves at least through autonomic nervous system (ANS). ANS at rest was found to be partially interlocked with activity of motor brain regions-the caudate nuclei and the motor cortex [14]. Magnetic stimulation of the human motor cortex evokes skin sympathetic nerve activity [15]. Transcutaneous electrical nerve stimulation (TENS), a maneuver used for pain control, has been found to influence cardiovascular responses through ANS reflex and to enhance motor function, visuospatial abilities, postural control, and cognitive function $[16,17]$. For example, sympathetic activity increased but parasympathetic activity decreased after flight simulator exposure [17] or during motion sickness (MS) evoked by the conflict among somatosensory, visual, and vestibular input [16], and TENS was effective in reducing the symptoms of MS [16] or simulator sickness developed during or after flight simulator training $[16,17]$ as well as alleviating cognitive impairment.

4.3. The Relationship between Gender and Visual Image Operation Level. There have been few studies on the comparison of the abilities of male and female in their visual image operation. It was pointed out that there were differences in the ability of spatial image, where the male outperformed the female [18]. This is interpreted as that the male outperformed the female in spatial perception, which leads to the male's advantage in spatial image operation based on it. But some researchers disagreed with this view. They held that image was not simply derivant of perception but was born out of symbolic mechanism or signal function $[10,19]$. Image is restricted at least by factors from two aspects: one is the objective material provided by perception and the other is the thinking pattern. So the content and form of image depend on the outside perceptive activities and then 
the internalized schema [20]. According to this viewpoint, it is not well grounded to attribute the performance difference in spatial image to the different levels of spatial perception solely. Findings from the present study showed that both the athletes have improved and developed in the visual image level and ability, but the difference in gender is not significant.

\subsection{Applications in Obsessive-Compulsive Disorder} Treatments. Obsessive-compulsive disorder (OCD) may be treated by phototherapy, but its therapeutic effects depended on its seasonality. The serotonin transporter (HTT) is a candidate gene for OCD that has been associated with anxiety-related traits. The long (l) and short (s) variants of the HTT promoter have different transcriptional efficiencies. Subjects with the $1 / 1$ and $1 / \mathrm{s}$ genotypes had significantly higher blood 5-hydroxytryptamine (5-HT) levels than those with the s/s genotype. There was a significant interaction between HTT promoter genotype and seasonal variation in blood 5-HT content, with significant seasonal differences in $5-\mathrm{HT}$ occurring only in the subjects with the $1 / 1$ genotype [21]. Yoney et al. [22] found that the patients with OCD did not report a greater degree of seasonal variations than normal and no response was seen to bright light therapy in the small number of patients treated. On the other hand, Höflich et al. [23] reported the case of a 40-year-old woman with a seasonal form of OCD which was usually accompanied by obsessions and occurred only in autumn or winter. After a 12-day treatment with full spectrum bright light (3000 lux; 2 hours a day between 9 and $11 \mathrm{am}$ ) without changing the long-term antidepressive medication $(125 \mathrm{mg}$ amitriptyline/day) there was a complete remission of OCD symptomatology, with no relapse during the next months.

OCD may also be treated by exercise therapy. Half of pediatric-onset OCD cases remit by adulthood, but visuospatial and fine-motor skill deficits are predictive of poor longterm outcome in pediatric-onset OCD [24]. This suggested that exercise may reduce OCD symptoms since our study suggested that fine-motor skill may promote visuospatial skill. Brown et al. [25] enrolled fifteen patients (53\% male; mean age $=44.4$ years) in a 12 -week moderate-intensity exercise intervention. Measures of OCD symptom severity were obtained at baseline, at end of treatment, and at 3- and 6-week and 6-month follow-up. They found a beneficial effect (Cohen's $d=1.69$ ) of a 12-week aerobic exercise intervention on reduction in OCD symptom severity. Abrantes et al. [26] further examined acute changes in OCD symptoms after engaging in single exercise sessions during a 12-week exercise intervention for 15 (53\% female; mean age $=41.9$ years) patients with OCD. Participants reported reductions in negative mood, anxiety, and OCD symptoms at the end of each exercise session relative to the beginning. Changes in the magnitude of the effect of exercise in reducing negative mood and anxiety remained fairly stable while levels of self-reported obsessions and compulsions decreased over the duration of the intervention. Results of this study point toward the promising effect of exercise for acute symptom reduction in patients with OCD.

\section{Conclusions}

There may be close relationship between sport skill level and the operation level of visual images. The higher the sport skill level is, the higher the operation level may be. The gymnasts may be faster in the operation of visual images than other athletes, but there may be no significant difference among athletes of other sports games in visual image operation. The athletes may achieve great improvement in their ability of visual image, but there may be no significant difference between different genders.

\section{Conflict of Interests}

The authors declare that there is no conflict of interests regarding the publication of this paper.

\section{Authors' Contribution}

P. Zeng and L. Liu contributed equally to this work.

\section{Acknowledgments}

This work was supported by National Science Foundation of China (60878061, 10974061, and 11374107), Doctoral Fund of Ministry of Education of China (20124407110013), and Guangdong Scientific Project (2012B031600004).

\section{References}

[1] J. Bo and R. D. Seidler, "Visuospatial working memory capacity predicts the organization of acquired explicit motor sequences," Journal of Neurophysiology, vol. 101, no. 6, pp. 3116-3125, 2009.

[2] J. Bo, V. Borza, and R. D. Seidler, "Age-related declines in visuospatial working memory correlate with deficits in explicit motor sequence learning," Journal of Neurophysiology, vol. 102, no. 5, pp. 2744-2754, 2009.

[3] M. W. G. Dye, C. S. Green, and D. Bavelier, "Increasing speed of processing with action video games," Current Directions in Psychological Science, vol. 18, no. 6, pp. 321-326, 2009.

[4] B. D. Glass, W. T. Maddox, and B. C. Love, "Real-time strategy game training: emergence of a cognitive flexibility trait," PLoS One, vol. 8, no. 8, Article ID e70350, 2013.

[5] N. Kida, S. Oda, and M. Matsumura, "Intensive baseball practice improves the Go/Nogo reaction time, but not the simple reaction time," Cognitive Brain Research, vol. 22, no. 2, pp. 257264, 2005.

[6] G. Giglia, F. Brighina, D. Zangla et al., "Visuospatial attention lateralization in volleyball players and in rowers," Perceptual and Motor Skills, vol. 112, no. 3, pp. 915-925, 2011.

[7] J. Seo, Y.-T. Kim, H.-J. Song et al., "Stronger activation and deactivation in archery experts for differential cognitive strategy in visuospatial working memory processing," Behavioural Brain Research, vol. 229, no. 1, pp. 185-193, 2012.

[8] M. Muiños and S. Ballesteros, "Visuospatial attention and motor skills in kung fu athletes," Perception, vol. 42, no. 10, pp. 1043-1050, 2013.

[9] A. B. Bhavanani, M. Ramanathan, R. Balaji, and D. Pushpa, "Immediate effects of Suryanamaskar on reaction time and 
heart rate in female volunteers," Indian Journal of Physiology and Pharmacology, vol. 57, no. 2, pp. 199-204, 2013.

[10] T. C. Y. Liu, R. Liu, L. Zhu, J. Q. Yuan, M. Wu, and S. H. Liu, "Homeostatic photobiomodulation," Frontiers of Optoelectronics in China, vol. 2, no. 1, pp. 1-8, 2009.

[11] T. C. Y. Liu, Y. Y. Liu, E. X. Wei, and F. H. Li, "Photobiomodulation on stress," International Journal of Photoenergy, vol. 2012, Article ID 628649, 11 pages, 2012.

[12] T. C. Y. Liu, L. Liu, J. G. Chen, P. Zeng, and X. B. Yang, "Actiondependent photobiomodulation on health, suboptimal health and disease," International Journal of Photoenergy, vol. 2014, Article ID 832706, 11 pages, 2014.

[13] M. T. Jensen, P. Suadicani, H. O. Hein, and F. Gyntelberg, "Elevated resting heart rate, physical fitness and all-cause mortality: a 16-year follow-up in the Copenhagen Male Study," Heart, vol. 99, no. 12, pp. 882-887, 2013.

[14] P. Schlindwein, H.-G. Buchholz, M. Schreckenberger, P. Bartenstein, M. Dieterich, and F. Birklein, "Sympathetic activity at rest and motor brain areas: FDG-PET study," Autonomic Neuroscience: Basic and Clinical, vol. 143, no. 1-2, pp. 27-32, 2008.

[15] D. H. Silber, L. I. Sinoway, U. A. Leuenberger, and V. E. Amassian, "Magnetic stimulation of the human motor cortex evokes skin sympathetic nerve activity," Journal of Applied Physiology, vol. 88, no. 1, pp. 126-134, 2000.

[16] H. Chu, M. H. Li, S. H. Juan, and W. Y. Chiou, "Effects of transcutaneous electrical nerve stimulation on motion sickness induced by rotary chair: a crossover study," Journal of Alternative and Complementary Medicine, vol. 18, no. 5, pp. 494-500, 2012.

[17] H. Chu, M. H. Li, Y. C. Huang, and S. Y. Lee, "Simultaneous transcutaneous electrical nerve stimulation mitigates simulator sickness symptoms in healthy adults: a crossover study, $B M C$ Complementary and Alternative Medicine, vol. 13, no. 1, article 84, 2013.

[18] W. Hidetoshi and Y. M. Chen, "Intelligence differences between men and women," Information on Psychological Sciences, vol. 6, pp. 56-58, 1981.

[19] R. H. Cox, Sport Psychology: Concepts and Applications, McGraw-Hall, 6th edition, 2007.

[20] Y. S. Lei, Comments on Epistomology of Piaget, People's Publishing House, Beijing, China, 1987.

[21] G. L. Hanna, J. A. Himle, G. C. Curtis et al., "Serotonin transporter and seasonal variation in blood serotonin in families with obsessive-compulsive disorder," Neuropsychopharmacology, vol. 18, no. 2, pp. 102-111, 1998.

[22] T. H. Yoney, T. A. Pigott, F. L'Heureux, and N. E. Rosenthal, "Seasonal variation in obsessive-compulsive disorder: preliminary experience with light treatment," The American Journal of Psychiatry, vol. 148, no. 12, pp. 1727-1729, 1991.

[23] G. Höflich, S. Kasper, and H. J. Möller, "Successful treatment of seasonal compulsive syndrome with phototherapy," Der Nervenarzt, vol. 63, no. 11, pp. 701-704, 1992.

[24] M. H. Bloch, D. G. Sukhodolsky, P. A. Dombrowski et al., "Poor fine-motor and visuospatial skills predict persistence of pediatric-onset obsessive-compulsive disorder into adulthood," Journal of Child Psychology and Psychiatry and Allied Disciplines, vol. 52, no. 9, pp. 974-983, 2011.

[25] R. A. Brown, A. M. Abrantes, D. R. Strong et al., "A pilot study of moderate-intensity aerobic exercise for obsessive compulsive disorder," Journal of Nervous and Mental Disease, vol. 195, no. 6, pp. 514-520, 2007.
[26] A. M. Abrantes, D. R. Strong, A. Cohn et al., "Acute changes in obsessions and compulsions following moderate-intensity aerobic exercise among patients with obsessive-compulsive disorder," Journal of Anxiety Disorders, vol. 23, no. 7, pp. 923927, 2009. 

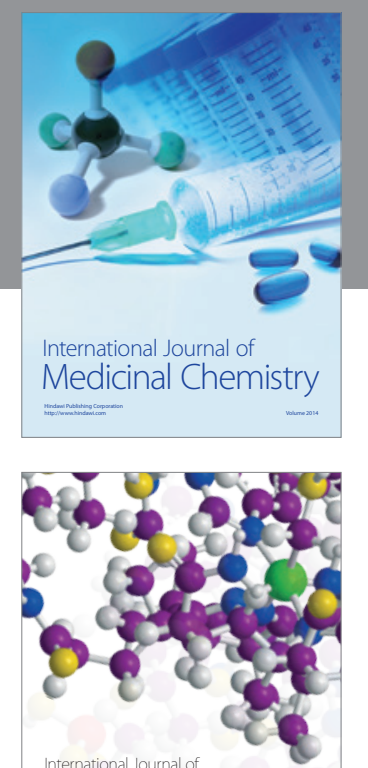

\section{Carbohydrate} Chemistry

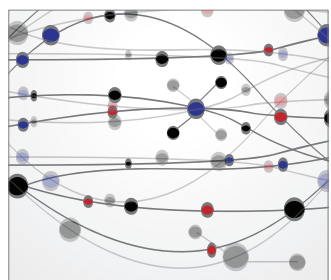

The Scientific World Journal
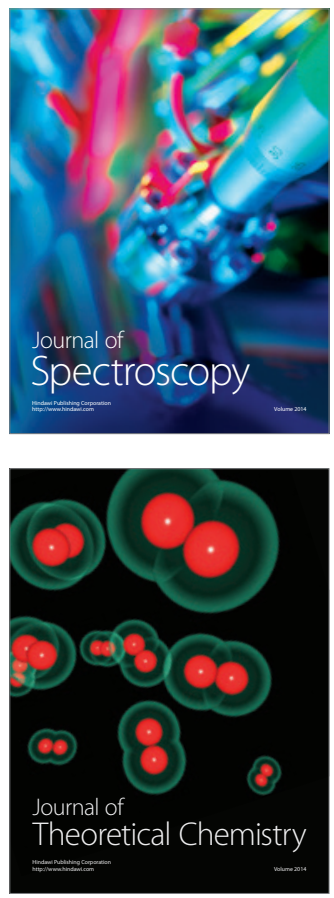
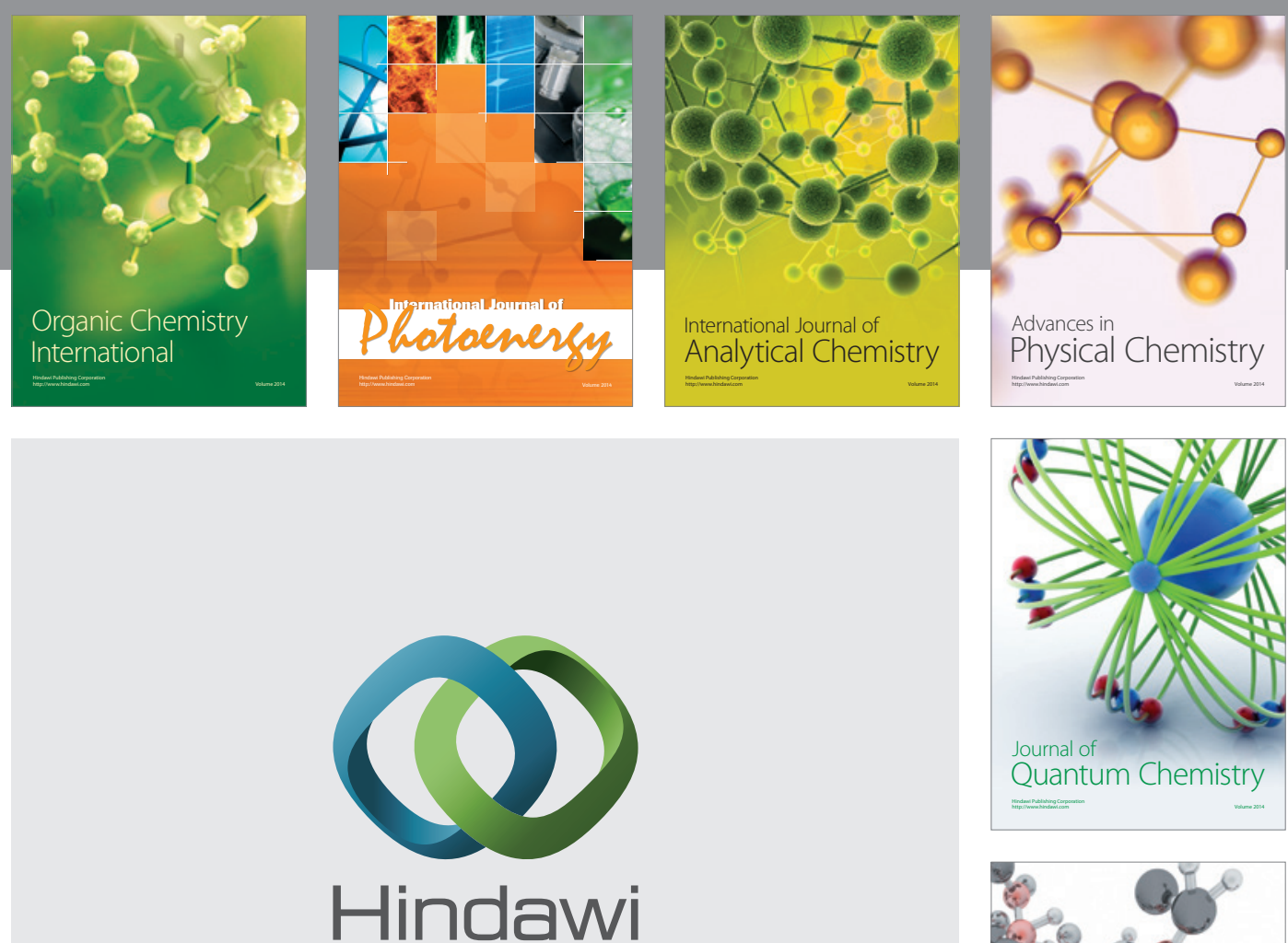

Submit your manuscripts at

http://www.hindawi.com

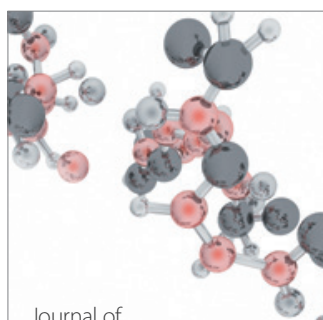

Analytical Methods

in Chemistry

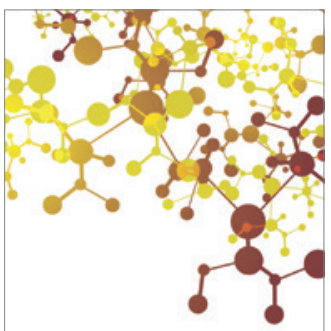

Journal of

Applied Chemistry

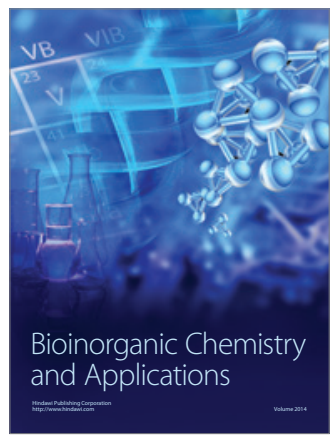

Inorganic Chemistry
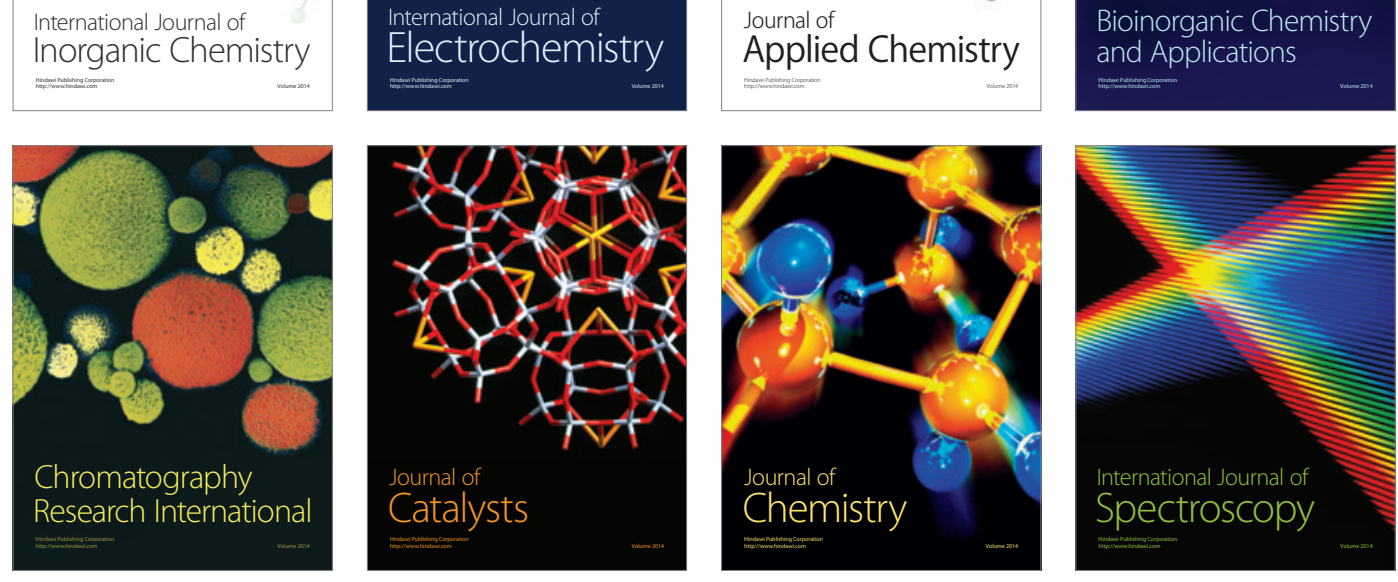